\title{
Sulfasalazine attenuates tamoxifen-induced toxicity in human retinal pigment epithelial cells
}

\author{
Narae Hwang \& Su Wol Chung ${ }^{*}$ \\ Department of Biological Sciences, University of Ulsan, Ulsan 44610, Korea
}

\begin{abstract}
Tamoxifen, a nonsteroidal estrogen receptor (ER) antagonist, is used routinely as a chemotherapeutic agent for ER-positive breast cancer. However, it is also causes side effects, including retinotoxicity. The retinal pigment epithelium (RPE) has been recognized as the primary target of tamoxifen-induced retinotoxicity. The RPE plays an essential physiological role in the normal functioning of the retina. Nonetheless, potential therapeutic agents to prevent tamoxifen-induced retinotoxicity in breast cancer patients have not been investigated. Here, we evaluated the action mechanisms of sulfasalazine against tamoxifen-induced RPE cell death. Tamoxifen induced reactive oxygen species (ROS)-mediated autophagic cell death and caspase-1mediated pyroptosis in RPE cells. However, sulfasalazine reduced tamoxifen-induced total ROS and ROS-mediated autophagic RPE cell death. Also, mRNA levels of tamoxifen-induced pyroptosis-related genes, IL-1 $\beta$, NLRP3, and procaspase-1, also decreased in the presence of sulfasalazine in RPE cells. Additionally, the mRNA levels of tamoxifen-induced AMD-related genes, such as complement factor I (CFI), complement factor H (CFH), apolipoprotein E (APOE), apolipoprotein J (APOJ), toll-like receptor 2 (TLR2) and toll-like receptor 4 (TLR4), were downregulated in RPE cells. Together, these data provide novel insight into the therapeutic effects of sulfasalazine against tamoxifen-induced RPE cell death. [BMB Reports 2020; 53(5): 284-289]
\end{abstract}

\section{INTRODUCTION}

Tamoxifen is a selective estrogen receptor (ER) modulator that acts as an anti-estrogen. Although the hormone estrogen promotes the growth of breast cancer cells, tamoxifen blocks estrogen from attaching to the ER on these cells (1). Thus, tamo-

${ }^{*}$ Corresponding author. Tel: +82-52-259-1641; Fax: +82-52-2591694; E-mail: swchung@ulsan.ac.kr

https://doi.org/10.5483/BMBRep.2020.53.5.041

Received 20 February 2020, Revised 6 March 2020, Accepted 10 April 2020

Keywords: Autophagic cell death, Pyroptosis, RPE, Sulfasalazine, Tamoxifen xifen decreases the chance of recurrence in some early-stage breast cancers and prevents cancer development in ER-positive breast cancer (1).

However, despite its effectiveness, tamoxifen is also associated with side effects, including blood clots, stroke, endometrial cancer, and ocular toxicity (2). Incidence of the ocular side effects of tamoxifen occurs $0.9 \%-11 \%$ and induces maculainvolving retinopathy, which can seriously compromise vision, especially in females treated with high daily or cumulative doses of tamoxifen (3). Also, tamoxifen leads to corneal and photoreceptor toxicity, progression of cataracts, retinopathy, and neuropathy (3). Although recent reports have described that tamoxifen toxicity impacts photoreceptors, the retinal pigment epithelium (RPE) is the primary target of tamoxifen toxicity (4). The role of RPE is critical for the survival of the retina, especially for maintaining the overlaying photoreceptors, phagocytizing the old photoreceptor outer segment, and mediating the uptake of nutrients, ions, and water (5). Furthermore, The most of retinal degenerative disease, such as age-related marcular degeneration (AMD), central serous retinopathy, and tamoxifen-induced retinopathy, are closely associated with RPE cell death (5).

Autophagy and inflammation-mediated pyroptosis are common features in tamoxifen-induced death of RPE cells (4). During autophagy, autophagosomes, which are double-membrane vesicles, engulf damaged proteins and organelles and then fuse with lysosomal vesicles (6). The core machinery of autophagy comprises more than 30 autophagy-related proteins, including LC3B. LC3B is a structural component of autophagosomes and is a standard marker for autophagosome (6). Although autophagy is necessary for common cellular functions, it also induces cell death under particular circumstances. Autophagic cell death has been implicated in ischemic brain injury, heart and liver diseases, and myopathies (7). Pyroptosis determines as an inflammation-mediated cell death caused by inflammasomes-induced activation of caspase-1 (8). Especially, autophagy and NLRP3 inflammasome are involved in tamoxifen-induced RPE cell death (4). However, despite this knowledge, there is currently no method or drug to prevent tamoxifen-induced RPE cell death in breast cancer patients undergoing tamoxifen therapy.

Sulfasalazine, a prodrug comprising 5-aminosalicylic acid (5-ASA) linked to sulfapyridine (SPD), was initially developed to treat rheumatoid arthritis (RA) (9), and is used to treat

ISSN: 1976-670X (electronic edition)

Copyright (c) 2020 by the The Korean Society for Biochemistry and Molecular Biology

(c) This is an open-access article distributed under the terms of the Creative Commons Attribution Non-Commercial License (http://creativecommons.org/licenses/by-nc/4.0) which permits unrestricted non-commercial use, distribution, and reproduction in any medium, provided the original work is properly cited. 
inflammatory bowel disease (IBD) and other autoimmune conditions. It acts by reducing inflammation (10). Although the role of sulfasalazine is different in many diseases, this orally applicable drug exerts anti-inflammatory and immunomodulatory efficacy (9). However, the impacts of sulfasalazine against tamoxifen-induced RPE cell death have not been investigated. Here, we investigated the cytoprotective effectsand mechanisms of sulfasalazine against tamoxifen-induced RPE cytotoxicity. The findings of this study may provide potential therapeutic implications for tamoxifen-induced retinal toxicity during tamoxifen treatment for breast cancer.

\section{RESULTS}

\section{Sulfasalazine inhibits tamoxifen-induced cell death in human RPE cells}

Several studies have shown that tamoxifen induces cytotoxicity and specifically impacts RPE cells (4). To investigate the impact of sulfasalazine on tamoxifen cytotoxicity, we first assessed the cytotoxicity of tamoxifen and sulfasalazine at various doses in RPE cells. Cell viability decreased after treatment with $10 \mu \mathrm{M}$ tamoxifen, and the decrease was more remarkable as tamoxifen concentrations increased (Fig. 1A). However, sulfasalazine exerted no cytotoxicity (Fig. 1A). Next, RPE cells were treated with vehicle, tamoxifen, or tamoxifen plus sulfasalazine at various doses for 24 hours (Fig. 1B). Tamoxifen-induced death of RPE cells was rescued by sulfasalazine, suggesting that sulfasalazine exerted protective effect against tamoxifen-induced RPE cell death.

We also investigated the expression of the apoptosis-related proteins caspase- 3 and cleaved caspase- 3 at various points after treatment with sulfasalazine, tamoxifen, or tamoxifen plus sulfasalazine (Fig. 1C). Sulfasalazine decreased the levels of cleaved caspase-3 in RPE cells, increased by tamoxifen.

Also, to identify metabolites of sulfasalazine responsible for its protective impacts on tamoxifen-induced RPE cell death, we treated RPE cells with vehicle, tamoxifen, tamoxifen plus sulfasalazine, 5-ASA, or SPD for 24 hours (Fig. 1D). Tamoxifeninduced cell death was rescued by the metabolites 5-ASA and SPD; however, the cytoprotective impacts of these metabolites were less potent than that of sulfasalazine. Interestingly, a combination of 5-ASA and SPD showed similar protective impacts of sulfasalazine on tamoxifen-induced RPE cell death. Additionally, sulfasalazine promoted tamoxifen-induced breast cancer cell death in MCF-7 cells (Fig. 1E). These data suggest that sulfasalazine specifically inhibited tamoxifen-induced RPE cell death.

\section{Sulfasalazine reduces tamoxifen-mediated ROS production in human RPE cells}

To identify the mediator molecules involved in tamoxifeninduced RPE cell death, total intracellular ROS and superoxide levels were measured after 12 hours of treatment with vehicle, sulfasalazine, tamoxifen, or tamoxifen, plus sulfasalazine using
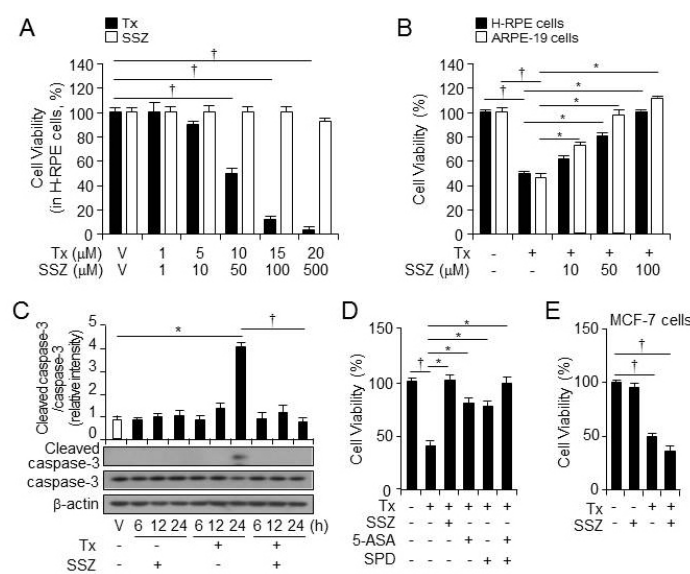

Fig 1. Sulfasalazine rescues tamoxifen-induced cell death in human RPE cells. (A) The cell viability of primary H-RPE cells $(n=12)$ was analyzed at 24 hours after the administration of various doses of tamoxifen (Tx) and sulfasalazine (SSZ). ${ }^{\top} \mathrm{P}<0.05$, decreased cell viability after treatment with tamoxifen vsvehicle. (B) The cell viability of primary H-RPE cells (black, $\mathrm{n}=12$ ) and ARPE-19 cells (white, $\mathrm{n}=$ 12) was analyzed at 24 hours after the administration of various doses of sulfasalazine in the presence of tamoxifen. ${ }^{\dagger} \mathrm{P}<0.05$, decreased cell viability after treatment with tamoxifen vs vehicle. ${ }^{*} \mathrm{P}<0.05$, increased cell viability after treatment with tamoxifen plus sulfasalazine vs tamoxifen only. (C) The caspase-3 and cleaved caspase- 3 expressions in the primary H-RPE cells were measured by western blotting at various points after vehicle, sulfasalazine $(100 \mu \mathrm{M})$, tamoxifen $(10 \mu \mathrm{M})$, or sulfasalazine plus tamoxifen treatment. Also, $\beta$-actin was used as a control for normalization. This blot is representative of the three independent experiments. Additionally, $* P<0.05$, increased protein levels of cleaved caspase-3 after treatment with tamoxifen vs vehicle. ${ }^{\dagger} \mathrm{P}<0.05$, decreased protein levels of cleaved caspase-3 after treatment with tamoxifen plus sulfasalazine vs tamoxifen only. (D) The cell viability of the primary H-RPE cells $(n=12)$ was analyzed after treatment with tamoxifen, tamoxifen plus sulfasalazine, tamoxifen plus 5-ASA $(100 \mu \mathrm{M})$, tamoxifen plus SPD $(100 \mu \mathrm{M})$, or tamoxifen plus 5-ASA and SPD. And, ${ }^{\dagger} \mathrm{P}$ $<0.05$, decreased cell viability after treatment with tamoxifen vs vehicle. ${ }^{*} \mathrm{P}<0.05$, increased cell viability after treatment with tamoxifen plus sulfasalazine, tamoxifen plus 5-ASA, tamoxifen plus SPD, or tamoxifen plus 5-ASA and SPD vs tamoxifen only. (E) The cell viability in MCF-7 breast cancer cells $(n=12)$ was analyzed after treatment with tamoxifen or tamoxifen plus sulfasalazine. ${ }^{\dagger} \mathrm{P}<$ 0.05 , decreased cell viability after treatment with tamoxifen vs vehicle. Values are presented as mean \pm SD.

flow cytometry. The total ROS and superoxide levels increased after tamoxifen administration; however, sulfasalazine decreased the tamoxifen-induced increase in total ROS and superoxide levels (Fig. 2A and 2B). Also, the ROS scavenger NAC rescued tamoxifen-induced RPE cell death in RPE cells (Fig. 2C). In contrast with these findings, tamoxifen-induced increase in the mRNA levels of antioxidant enzymes was not rescued by sulfasalazine (Fig. 2D-2I). These data suggest that sulfasalazine decreased tamoxifen-induced increase in total ROS and superoxide levels, and the cytoprotective effects of sulfasalazine in RPE cells may not be related with the mRNA expression of 
A

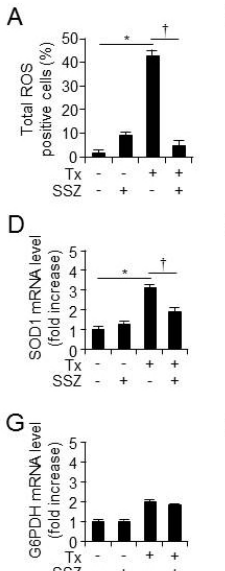

$\mathrm{B}$
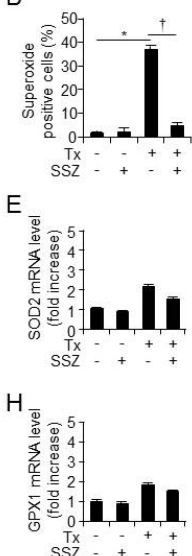
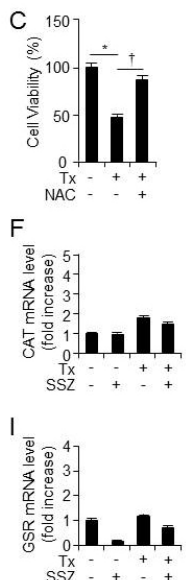

Fig. 2. Sulfasalazine inhibits tamoxifen-induced ROS in human RPE cells. ARPE-19 cells were treated with tamoxifen or tamoxifen plus sulfasalazine for 12 hours. The total $\operatorname{ROS}(A, n=12)$ and superoxide $(B, n=12)$ levels were measured by flow cytometry. And, $* P<0.05$, increased ROS level after treatment with tamoxifen vs vehicle. ${ }^{\dagger} P<0.05$, decreased ROS level after treatment with tamoxifen plus sulfasalazine vs tamoxifen only. (C) The cell viability was analyzed at 24 hours after tamoxifen treatment in the presence or absence of NAC $(10 \mathrm{mM})$. Also, ${ }^{\dagger} P<0.05$, decreased cell viability after treatment with tamoxifen vs vehicle. Additionally, ${ }^{*} P<0.05$, increased cell viability after treatment with tamoxifen plus NAC vs tamoxifen only. The mRNA levels of the antioxidant enzymes SOD1 (D), SOD2 (E), CAT (F), G6PDH (G), GPX2 (H), and GSR (I) in primary H-RPE cells $(n=3)$ were assessed at 12 hours after treatment with vehicle, tamoxifen, sulfasalazine, or tamoxifen plus sulfasalazine. And, $* \mathrm{P}<0.05$, increased the mRNA levels of genes after treatment with tamoxifen vs vehicle. Also, ${ }^{+} \mathrm{P}<0.05$, decreased the mRNA levels of genes after treatment with tamoxifen plus sulfasalazine vs tamoxifen only. Values are presented as mean $\pm \mathrm{SD}$.

antioxidants enzymes.

\section{Sulfasalazine diminishes tamoxifen-mediated autophagic cell death in human RPE cells}

Recent studies have suggested that autophagy is involved in tamoxifen-induced cell death (4); additionally, we previously reported that enhanced ROS leads to autophagy (11). To confirm if tamoxifen induces autophagy in RPE cells, we treated RPE cells with vehicle, sulfasalazine, tamoxifen, or tamoxifen plus sulfasalazine. The tamoxifen treatment increased the levels of LC3B-II (the lower band of LC3B) protein, whereas sulfasalazine treatment blocked autophagy (Fig. 3A and 3B). Also, the tamoxifen-induced decrease in cell viability was rescued by an autophagy inhibitor (Baf-1) (Fig. 3C). To further investigate if tamoxifen-induced ROS production triggers autophagy, we treated RPE cells with vehicle, tamoxifen, or tamoxifen plus NAC, and we harvested total RNA and protein after cell treatment (Fig. 3D and 3E). Tamoxifen increased the mRNA and protein levels of LC3B-II (the lower band of LC3B), whereas the ROS scavenger (NAC) decreased the mRNA and
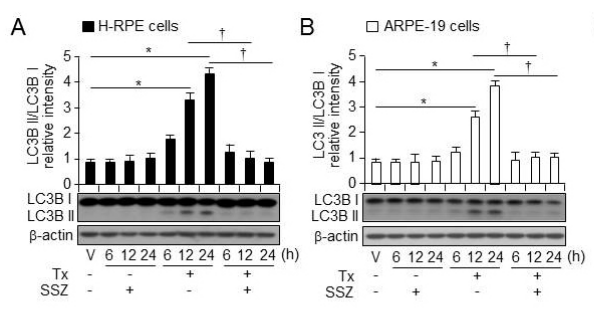

D
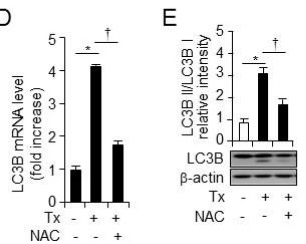

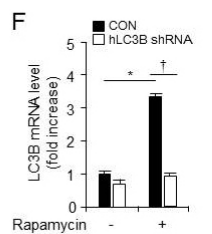

C

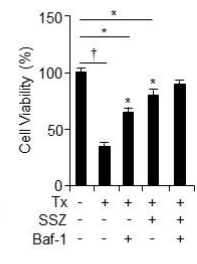

Fig. 3. Sulfasalazine decreases tamoxifen-induced autophagic cell death in human RPE cells. LC3B I and II expression in the primary $\mathrm{H}$-RPE cells $(\mathrm{A}, \mathrm{n}=3)$ and ARPE-19 cells $(\mathrm{B}, \mathrm{n}=3)$ was assessed by western blotting at various points after vehicle, sulfasalazine, tamoxifen,or tamoxifen plus sulfasalazine administration. Also, $\beta$-actin was used as a control for normalization. This blot is representative of the three independent experiments. Also, $* P<0.05$, increased ratio of $\mathrm{LC} 3 \mathrm{II} / \mathrm{I}$ protein levels after treatment with tamoxifen vs vehicle. ${ }^{\dagger} \mathrm{P}<0.05$, decreased ratio of $\mathrm{LC} 3 \mathrm{II} / \mathrm{I}$ protein levels after treatment with tamoxifen plus sulfasalazine vs tamoxifen only. (C) The cell viability of the primary H-RPE cells $(n=12)$ was analyzed at 24 hours after treatment with vehicle, tamoxifen, tamoxifen plus an autophagy inhibitor (100 nM Baf-1), or tamoxifen plus sulfasalazine in the absence or presence of Baf- 1 . Additionally, ${ }^{\dagger} \mathrm{P}<0.05$, decreased cell viability after treatment with tamoxifen vs vehicle. ${ }^{*} P<0.05$, increased cell viability after treatment with vs tamoxifen only. (D) The mRNA levels of LC3B in the primary H-RPE cells $(n=3)$ were assessed at 12 hours after treatment with vehicle, tamoxifen, or tamoxifen plus NAC. $* \mathrm{P}<0.05$, increased mRNA levels of LC3B after treatment with tamoxifen $v s$ vehicle. ${ }^{\dagger} \mathrm{P}<0.05$, decreased mRNA levels of LC3B after treatment with tamoxifen plus NAC vs tamoxifen only. (E) The LC3B I and II expression in the primary H-RPE cells $(n=3)$ was assessed by western blotting at 24 hours after administration of vehicle, tamoxifen, tamoxifen plus NAC. And, $\beta$-actin was used as a control for normalization. This blot is representative of the three independent experiments. And, $* \mathrm{P}<0.05$, increased ratio of LC3 II/I protein levels after treatment with tamoxifen vs vehicle. ${ }^{\dagger} \mathrm{P}<0.05$, decreased ratio of LC3 II/I protein levels after treatment with tamoxifen plus NAC vs tamoxifen only. (F) The mRNA levels of LC3B were analyzed in control and the LC3B shRNA-expressing cells at 12 hours after vehicle or rapamycin $(1 \mu \mathrm{M})$ treatment. Additionally, $* P<0.05$, increased the mRNA levels of LC3B after treatment with rapamycin vs vehicle. ${ }^{\dagger} \mathrm{P}<0.05$, decreased the mRNA levels of the LC3B in LC3B shRNA-expressing cells $v s$ in control shRNA-expressing cells. (G) The cell viability of the control or LC3B shRNA-expressing cells was analyzed at 24 hours after the administration of vehicle, tamoxifen, or tamoxifen plus sulfasalazine. ${ }^{*} \mathrm{P}<0.05$, increased cell viability after tamoxifen administration in LC3B shRNA-expressing cells vs in control shRNA-expressing cells. Too, $* * P<0.05$, increased cell viability after treatment with tamoxifen plus sulfasalazine vs tamoxifen only in the control and LC3B shRNA-expressing cells. Values are presented as mean $\pm S D, n=3$. 
protein levels of LC3B in RPE cells. These data suggest that tamoxifen-induced ROS triggers autophagy.

Thereafter, to investigate the mechanism of autophagy in tamoxifen-induced RPE cell death, we generated the LC3B and control shRNA-expressing cells. To examine the difference in the LC3B expression between the cells expressing LC3B shRNA and the control shRNA, the total RNA was harvested and the LC3B mRNA levels were analyzed after rapamycin treatment. The mRNA levels of the LC3B decreased in the LC3B shRNAexpressing cells, compared with those in the control shRNAexpressing cells (Fig. 3F).

To further investigate the critical role of autophagy in tamoxifeninduced RPE cell death, we assessed the viability of the LC3B orthe control shRNA-expressing cells after vehicle, sulfasalazine, tamoxifen, or tamoxifen plus sulfasalazine treatment (Fig. 3G). After the tamoxifen treatment, the degree of cell death was much lower in the LC3B shRNA-expressing cells than in the control shRNA-expressing cells. However, sulfasalazine reduced tamoxifen-induced RPE cell death in the LC3B and the control shRNA-expressing cells. These data suggest that tamoxifen-induced ROS production is involved in autophagy induction, and that reduction of tamoxifen-induced autophagy is partially involved in the impact of sulfasalazine.

\section{Sulfasalazine reduces tamoxifen-induced pyroptosis and AMD-associated gene expression}

Sulfasalazine is a well-known anti-inflammatory drug for the treatment of RA and IBD (9). A recent study showed that activated caspase-1-mediated pyroptosis is involved in tamoxifeninduced RPE cell toxicity (4). To investigate the anti-inflammatory effects of sulfasalazine, RPE cells were treated with vehicle, sulfasalazine, tamoxifen, or tamoxifen plus sulfasalazine, and the total RNA was extracted at 12 hours after cell treatment. Thereafter, the mRNA levels of IL-1 $\beta$, NLRP3, ASC, and caspase- 1 were measured using quantitative reverse transcription (qRT)-PCR (Fig. 4A-4D). Sulfasalazine decreased tamoxifeninduced mRNA levels of IL-1 $\beta$, NLRP3, and caspase- 1 in RPE cells; however, the mRNA levels of ASC did not change in the presence of sulfasalazine. To verify if the inhibition of caspase-1 is involved in the impacts of sulfasalazine on tamoxifeninduced RPE cell death, we treated the RPE cells with vehicle, tamoxifen, a caspase-1 inhibitor (A-YVAD-FMK), tamoxifen plus sulfasalazine, or tamoxifen plus caspase-1 for 24 hours (Fig. 4E). Tamoxifen-induced cell death was rescued by the caspase-1 inhibitor; however, sulfasalazine reduced tamoxifen-induced the RPE cell death in the RPE cells pretreated with caspase-1 inhibitor. These data suggest that sulfasalazine suppresses caspase-1-mediated inflammation and the death of RPE cellsin the presence of tamoxifen. Also, to examine if sulfasalazine decreases the mRNA levels of AMD-associated genes in response to tamoxifen in the RPE cells, we assessed the mRNA levels of AMD-associated genes, including $\mathrm{CFI}, \mathrm{CFH}, \mathrm{APOE}, \mathrm{APO}$, TLR2, and TLR4 (Fig. 4F-4K). The RPE cells were treated with vehicle, sulfasalazine, tamoxifen, or tamoxifen plus sulfasala-

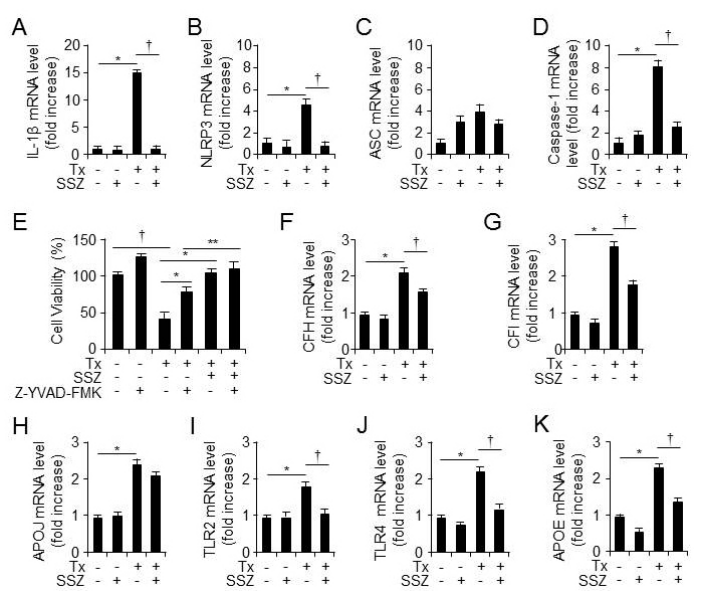

Fig. 4. Sulfasalazine reduces tamoxifen-induced mRNA levels of IL-1ßand AMD-associated genes in human RPE cells. The mRNA levels of IL-1 $\beta$ (A), NLRP3 (B), ASC (C), and procaspase-1 (D) in primary $\mathrm{H}-\mathrm{RPE}$ cells wereassessed at 12 hours after vehicle, tamoxifen, sulfasalazine, or tamoxifen plus sulfasalazine administration. Also, $* P<0.05$, increased the mRNA levels of genes after treatment with tamoxifen vs vehicle. ${ }^{\dagger} \mathrm{P}<0.05$, decreased the mRNA levels of genes after treatment with tamoxifen plus sulfasalazine vs tamoxifen only $n=3$. (E) The cell viability of the primary H-RPE cells ( $\mathrm{n}=12$ ) was analyzed at 24 hours after the administration of vehicle, tamoxifen, tamoxifen plus the caspase- 1 inhibitor Z-YVADFMK $(20 \mu \mathrm{M})$, or tamoxifen plus sulfasalazine in the absence or presence of Z-YVAD-FMK. Additionally, ${ }^{+} P<0.05$, decreased cell viability after treatment with tamoxifen vs vehicle. ${ }^{* P}<0.05$, increased cell viability after treatment with tamoxifen plus sulfasalazine or Z-YVAD-FMK vs tamoxifen only. The mRNA levels of $\mathrm{CFH}(\mathrm{F}), \mathrm{CFI}(\mathrm{G}), \mathrm{APOJ}(\mathrm{H}), \operatorname{TLR} 2(\mathrm{I}), \mathrm{TLR} 4(\mathrm{~J})$, and APOE $(\mathrm{K})$ in the primary H-RPE cells $(n=3)$ were assessed at 12 hours after the administration of vehicle, tamoxifen, sulfasalazine, or tamoxifen plus sulfasalazine. Also, ${ }^{*} \mathrm{P}<0.05$, increased the mRNA levels of genes after treatment with tamoxifen $v s$ vehicle. ${ }^{\dagger} \mathrm{P}<0.05$, decreased the mRNA levels of genes after treatment with tamoxifen plus sulfasalazine vs tamoxifen only. Values are presented as mean $\pm \mathrm{SD}$.

zine, and total RNA was extracted at 12 hours after administration (Fig. 4F-4K). The sulfasalazine decreased tamoxifeninduced increase in the mRNA levels of AMD-associated genes in the RPE cells. These results suggest that sulfasalazine decreases tamoxifen-induced pyroptosis and increases in the mRNA levels of AMD-associated gene in the RPE cells.

\section{DISCUSSION}

In this study, we confirmed that tamoxifen induced cell death and elevated the level of cleaved caspase-3, a pro-apoptosis protein, in the RPE cells (Fig. 1A and 1C), and examined the therapeutic potential and molecular mechanisms of sulfasalazine against tamoxifen-induced RPE cell death. As shown in Fig. 1B, sulfasalazine rescued tamoxifen-induced RPE death of the RPE cells, but not that of the MCF-7 breast cancer cells 
(Fig. 1E); in fact, the sulfasalazine increased MCF-7 cell death. Sulfapyridine is responsible for many of the side effects of sulfasalazine, whereas 5-ASA is responsible for many of its beneficial effects of sulfasalazine in patients with IBD (9). However, 5-ASA and sulfapyridine showed similar beneficial effects, and a combination of both drugs, similar to sulfasalazine, fully recovered tamoxifen-induced RPE cell death (Fig. 1D). These data suggest that sulfasalazine may be a potential drug for preventing ocular toxicity in breast cancer patients undergoing tamoxifen therapy.

Next, we investigated the molecular mechanisms of sulfasalazine in alleviating tamoxifen-induced RPE cell death. Recent studies have shown that tamoxifen-induced ROS and autophagic cell death are involved in tamoxifen toxicity in the ARPE cells (4). Also, we previously reported that enhanced ROS induces autophagic cell death (11). Sulfasalazine decreased tamoxifeninduced increases in total ROS and superoxide levels, and an ROS scavenger decreased tamoxifen-induced RPE cell death (Fig. 2A-2C). However, sulfasalazine did not show antioxidant effects in the RPE cells (Fig. 2D-2I). Tamoxifen-enhanced ROS production is involved in tamoxifen-induced autophagic cell death (Fig. 3). Autophagy is a stress-responsive survival mechanism. However, evidence of autophagic cell death has been (7). Tamoxifen-induced autophagy also contributes to cell death mechanisms, not survival mechanism, and tamoxifen is structurally similar to other drugs with well-known retinal impacts, including chloroquine (4). These data suggest that sulfasalazine may exert beneficial impacts on the retinotoxicity of chloroquine. Specifically, chloroquine, a well-known autophagy inhibitor, disrupts the lysosomal function and the phagocytosis in RPE cells. Although autophagy is involved in cell death mechanisms, the complete inhibition of autophagy may produce detrimental effects oncells. Interestingly, tamoxifen-induced RPE cell death was partially recovered in the LC3B shRNAexpressing cells (Fig. 3G). However, sulfasalazine fully rescued the viability of the LC3B shRNA-expressing cells compared with that of the control shRNA-expressing cells (Fig. 3G). These data suggest that sulfasalazine prevents tamoxifen-induced RPE cell death via another mechanism besides autophagic cell death.

Sulfasalazine is an anti-inflammatory drug (9), and tamoxifen induces pyroptosis, an inflammation-mediated programmed cell death (7). In the process of pyroptosis, cleaved caspase-1 is a key factor that mediates the massive generation of the pro-inflammatory cytokines IL-1 $\beta$ and IL-18, leading to cell death similar to apoptosis (8). As we expected, sulfasalazine decreased the tamoxifen-induced expression of IL-1 $\beta$, NLRP3, and caspase- 1 in the RPE cells (Fig. 4A-4D). Additionally, a caspase-1 inhibitor disrupted the impacts of sulfasalazine against tamoxifen-induced RPE cell death (Fig. 4E). These findings indicate that sulfasalazine prevents tamoxifen-induced RPE cell death by inhibiting autophagic cell death and pyroptosis.

Also, RPE cell death and dysfunction play a key role in the development of AMD, a side effect of tamoxifen (4), and sulfasalazine decreases the tamoxifen-induced expression of AMD-associated genes in the RPE cells (Fig. 4F-4K). These data suggest that sulfasalazine reduces tamoxifen-induced AMD.

In conclusion, this study showed that sulfasalazine exerts beneficial impacts against tamoxifen-induced RPE cell death by reducing ROS-mediated autophagic cell death and pyroptosis, revealing its potential as an effective drug preventing tamoxifen side effects during breast cancer therapy.

\section{MATERIALS AND METHODS}

\section{Cell culture and reagents}

Primary human fetal RPE (H-RPE) cells were purchased at passage one from LONZA (Walkersville, MD, USA), and all experiments were performed with cells at passage two to six. The ARPE-19 human RPE cells were purchased from the ATCC (Manassas, VA, USA). Tamoxifen and 5-ASA were purchased from Enzo Life Sciences (Farmingdale, NY, USA). The rapamycin was purchased from EMD Millipore Corporation (USA). Sulfasalazine, sulfapyridine, bafilomycin $A 1$, and other reagents were purchased from Sigma-Aldrich (St. Louis, MO, USA). NAC (Sigma-Aldrich) and Z-YVAD-FMK (BioVision Inc. Milpitas, $\mathrm{CA})$ were used as inhibitor reagents.

\section{Western immunoblotting}

Western immunoblotting was performed as previously described (12). Caspase-3 and cleaved caspase- 3 were obtained from Cell Signaling Technology (Danvers, MA), whereas the LC3B and $\beta$-actin were obtained from Sigma-Aldrich. The blots were stained with horseradish peroxidase-conjugated IgG and visualized with a SuperSignal West Pico Chemiluminescent Substrate (Pierce, Rockford, IL, USA).

\section{qRT-PCR}

Total RNA was isolated from the RPE cells using Trizol reagent (Invitrogen, Carlsbad, CA) and reverse transcribed to cDNA using a SuperScript ${ }^{\mathrm{TM}}$ III First-Strand Synthesis System (Invitrogen). In this study, qPCR was conducted using iQ SYBR Green Supermix (Bio-Rad). The primer sequences for G6PDH, GSR, GPX1, SOD1, SOD2, CAT, CFH, CFI, APOE, APOJ, TLR2, and TLR4 were described previously (13). The primers used were as follow: human LC3B (5'-ACC ATG CCG TCG GAG AAG-3'and 5'ATC GTT CTA TTA TCA CCG GGA TTT T-3', IL-1 $\beta$ (5'AAA TAC CTG TGG CCT TGG GC-3' and 5'-TTT GGG ATC TAC ACT CTC CAG CT-3', NLRP3 (5'TGA AGA AAG ATT ACC GTA AGA AGT ACA GA-3'nd 5'GCG TTT GTT GAG GCT CAC ACT-3', ASC (5'GT TTC ACA CCA GCC TGG AA-3' and 5'-TTT TCA AGC TGG CTT TTC GT-3'), Caspase-1 (5'-CAT CAC AGG CAT GAC AAT GCT GCT-3' and $5^{\prime}$-TGC CTT CCC GAA TAC CAT GAG ACA- $\left.3^{\prime}\right)$, and $\beta$-actin (5'-ATC GTG CGT GAC ATT AAG GAG AAG-3' and 5'-AGG AAG GAA GGC TGG AAG AGT G-3'). 


\section{Construction of LC3B shRNA-expressing cells}

The LC3B shRNA and the nonspecific control shRNA (SigmaAldrich) were transfected into ARPE-19 cells using transfection reagents (Promega, Madison, WI, USA) according to the manufacturer's protocol. The sequences of human LC3B shRNA were as follow: 5'-CCG GGT TCG GGA TGA AAT TGT CAG TCT CGA GAC TGA CAA TTT CAT CCC GAA CTT TTT TG-3'. The expression of LC3B and $\beta$-actin in stable cells was measured.

\section{Cell viability assay}

Cell viability was determined by the MTS assay using a Cell Titer 96 AQueous one solution cell proliferation assay kit (Promega) according to the protocol of the manufacturer.

\section{ROS detection assay}

The ROS levels were determined by using a ROS-ID ${ }^{\mathbb{R}}$ Total ROS/Superoxide Detection Kit (Enzo Life Sciences, Farmingdale, NY, USA) according to the manufacturer's protocol. Positive cells and total ROS levels were measured using a FACS Calibur flow cytometer (Becton Dickinson, San Jose, CA, USA).

\section{Statistical analysis}

The data represent mean \pm SD. For comparisons between the two groups, the two-tailed unpaired Student's $t$-test was used. For comparisons of timed series experiments, the paired Student's $t$-test was used. The Mann-Whitney $U$ test was performed to compare the mRNA expression of genes between the control and the LC3B shRNA-expressing cells. Statistically significant differences were accepted at $\mathrm{P}<0.05$.

\section{ACKNOWLEDGEMENTS}

This study was supported by the Basic Science Research Program through the National Research Foundation of Korea (NRF), funded by the Ministry of Education (NRF-2014R1A6A1030 318 to S.W.C.).

\section{CONFLICTS OF INTEREST}

The authors have no conflicting interests.

\section{REFERENCES}

1. Xiong R, Zhao J, Gutgesell LM et al (2017) Novel SERDs Developed against treatment-resistant breast cancer. J Med Chem 60, 1325-1342

2. Yang G, Nowsheen S, Aziz K and Georgakilas AG (2013) Toxicity and adverse effects of tamoxifen and other anti-estrogen drugs. Pharmacol Ther 139, 392-404

3. Wang L, Miao H and Li X (2015) Tamoxifen retinopathy: a case report. Springerplus 4, 501

4. Kim LA, Amarnani D, Gnanaguru G et al (2014) Tamoxifen toxicity in cultured RPE cells is mediated by concurrent regulated cell death mechanisms. Invest Ophthalmol Vis Sci 55, 4747-4758

5. Koulisis N, Moysidis SN, Olmos de Koo LC et al (2016) The tipping point: Tamoxifen toxicity, central serous chorioretinopathy, and the role of estrogen and its receptors. Am J Ophthalmol Case Rep 3, 8-13

6. Abdollahzadeh I, Hendriks J, Sanwald JL et al (2019) Autophagy-related proteins GABARAP and LC3B label structures of similar size but different shape in superresolution imaging. Molecules 24, 1833

7. Uchiyama Y, Shibata M, Koike M et al (2008) Autophagyphysiology and pathophysiology. Histochem Cell Biol $129,407-420$

8. Bergsbaken T, Fink SL and Cookson BT (2009) Pyroptosis: host cell death and inflammation. Nat Rev Microbiol 7, 99-109

9. Kümmerle-Deschner J and Dannecker G (1995) Sulphasalazine desensitization in a paediatric patient with juvenile chronic arthritis. Acta Paediatr 84, 952-954

10. Fakhoury M, Negrulj R, Mooranian A, Al-Salami H (2014) IBD: clinical aspects and treatments. J Inflamm Res 7, 113120

11. Park E and Chung SW (2019) ROS-mediated autophagy increases intracellular iron levels and ferroptosis by ferritin and transferrin receptor regulation. Cell Death Dis 10, 822

12. Kwon MY, Hwang N, Lee SJ and Chung SW (2019) NOD2 attenuates ER stress-induced cell death in VSMCs. BMB Rep 52, 665-670

13. Hwang N, Kwon MY, Woo JM and Chung SW (2019) Oxidative stress-induced pentraxin 3 expression human RPE cells is involved in the pathogenesis of AMD. Int J Mol Sci 20, 6028 